Discussion Paper No. 690

\title{
BELIEF UPDATING IN INDIVIDUAL AND SOCIAL LEARNING: \\ A FIELD EXPERIMENT ON THE INTERNET
}

\author{
Shunichiro Sasaki \\ and \\ Toshiji Kawagoe
}

May 2007

The Institute of Social and Economic Research Osaka University

6-1 Mihogaoka, Ibaraki, Osaka 567-0047, Japan 


\title{
Belief updating in individual and social learning:
}

\section{A field experiment on the Internet}

\author{
Shunichiro Sasaki ${ }^{\text {a,**}}$, Toshiji Kawagoe ${ }^{\mathrm{b}}$ \\ ${ }^{a}$ Institute of Social and Economic Research, Osaka University, \\ 6-1 Mihogaoka, Osaka 567-0047, Japan \\ ${ }^{\mathrm{b}}$ Future University - Hakodate, \\ 116-2 Kamedanakanocho, Hakodate, Hokkaido 041-8655, Japan
}

\begin{abstract}
We conducted a field experiment on the Internet and investigated the participants' belief updating in an individual learning environment where they observe a sequence of private signals and in a social learning environment where they observe a sequence of other people's actions. We observed that participants do not update their posterior beliefs as efficiently as Bayesian, and that participants rely more on private signals than on other people's actions even when the informativeness of both is identical. Furthermore, we confirmed that participant's trust in other people's actions and their conformity to other people's actions are affected by their demographic characteristics.
\end{abstract}

JEL Classification Codes: C93; D83

Keywords: Belief updating, individual learning, social learning, field experiment, conformity

\footnotetext{
* Corresponding author. Phone: +81-6-6877-5111 (ex. 9179), Fax: +81-6-6878-2766

Email addresses: ssasaki@iser.osaka-u.ac.jp (S. Sasaki), kawagoe@fun.ac.jp (T. Kawagoe)
} 


\section{Introduction}

We conducted a field experiment on the Internet and investigated how participants update their posterior beliefs on the underlying state of the world in an individual learning environment, where they can observe a sequence of private signals, and in a social learning environment, where they can observe a sequence of other people's actions. We analyzed whether participants' belief-updating behaviors are consistent with Bayesian theory and whether they differ depending on the learning environment, comparing with the results of the previous laboratory experiments on informational cascades. In addition, by measuring participant's degree of trust in other people's actions and their conformity to other people's actions, we examine whether they are affected by participant's demographic characteristics.

We observed that participants do not make use of private signals and other people's actions as efficiently as Bayesian theory assumes. We also found that participants report higher posterior beliefs in an individual learning environment than in a social learning environment, even when the theoretical informativeness of the observed sequence of private signals and other people's actions is identical. In addition, we confirmed that participant's trust in other people's actions and their conformity to other people's actions are affected by some of their demographic characteristics.

In the following section, we outline the field experiment and show the framework of individual and social learning. In Section 3, we present the behavioral hypotheses derived from Bayesian theory. In Section 4, we examine whether participants' belief-updating behaviors are consistent with the behavioral hypothesis and whether they differ depending on the learning environment. In Sections 5 and 6, we measure participant's degree of trust in other people's actions and their conformity to other 
people's actions and investigate whether they are affected by their demographic characteristics. In Section 7, we conclude the discussion.

\section{Individual and social learning in the field experiment}

The field experiment was conducted from February 23 to February 24, 2007, for the registered monitors of 'goo research', a polling agency in Japan. The monitors' ages ranged from 20 to 49 and their various occupations were (1) managers in a private company, (2) employees in an administrative position, (3) teachers, (4) lawyers, CPAs, and tax accountants, or (5) students in junior colleges, universities, or graduate schools ${ }^{1}$. The field experiment was conducted on the Internet. We sent emails that notified them of the URL at which the experiment would be conducted and 1033 monitors participated in the experiment ${ }^{2}$. When they logged onto the web site, they were randomly categorized into four groups $G \in\{1,2,3,4\}$.

The following situation related to decision making under uncertainty was described to the participants in the experiment. There are two states of the world $\omega \in\{A, B\}$ and each state is realized with the commonly known priors $\operatorname{Pr}(A)=\operatorname{Pr}(B)=1 / 2$. Participants do not know which state will be realized. However, they can infer the state of the world by observing either a 'private signal' $\sigma(\omega)$ of which preciseness is $\operatorname{Pr}(\sigma(\omega) \mid \omega)=2 / 3$ or other people's predictions $\pi(\omega)$. The role of participant $i$ in group $G$ in this decision-making problem is to submit a prediction about which state will be realized

\footnotetext{
${ }^{1}$ For details of participants' demographic characteristics, see Table 1.

2 They were paid 50 points (equivalent to 50 Japanese yen), which can be pooled and can be exchanged for a cash voucher in payment for participation.
} 
$\Pi(\omega)_{i, G}^{t}$ and its subjective posterior probability $\mu(A)_{i, G}^{t}$ and $\mu(B)_{i, G}^{t}=1-\mu(A)_{i, G}^{t}$ in round $t=1, \ldots, n$ based on observed sequences of private signals or other people's predictions.

We define the process of this decision-making problem as individual learning or social learning depending on the difference in information they observe as follows.

In the individual learning environment, participant $i$ in group $G$ submits $\Pi(\omega)_{i, G}^{t}, \mu(A)_{i, G}^{t}$ and $\mu(B)_{i, G}^{t}=1-\mu(A)_{i, G}^{t}$ after observing the sequence of private signals $\sigma(\omega)$ for $n=G+1$ rounds. In round $t<n$, participant $i$ in group $G$ faces a question $Q A_{G}^{t}$, which presents the private signal $\sigma(A)^{t}$ and asks the participant to submit $\Pi(\omega)_{i, G}^{t}$ and $\mu(A)_{i, G}^{t}{ }^{3}$. Thus, by question $Q A_{G}^{t}$, the sequences of private signals that participants in groups 1, 2, 3, and 4 observed from all rounds except the final round were $\left(\sigma(A)^{1}\right), \quad\left(\sigma(A)^{1}, \sigma(A)^{2}\right), \quad\left(\sigma(A)^{1}, \sigma(A)^{2}, \sigma(A)^{3}\right), \quad$ and $\left(\sigma(A)^{1}, \sigma(A)^{2}, \sigma(A)^{3}, \sigma(A)^{4}\right)$, respectively ${ }^{4}$. We name these sequences $S A_{1}, S A_{2}, S A_{3}$, and $S A_{4}$. On the other hand, in the final round $t=n$, participant $i$ in group $G$ faces a question $Q A_{G}^{n}$, which presents the private signal $\sigma(B)^{n}$ and asks the participant to submit $\Pi(\omega)_{i, G}^{n}$ and $\mu(A)_{i, G}^{n}$. Thus, by questions $Q A_{G}^{t}$ and $Q A_{G}^{n}$, the sequences of

\footnotetext{
${ }^{3}$ Although participants were told that the computer program would automatically generate hints $\sigma(A)$ or $\sigma(B)$ in each round based on the realized state, we presented a predetermined sequence of hints because we wanted to set up a situation where all the participants in each group observed the same sequence of hints.

${ }^{4}$ In round $t$, participants could also refer to the sequence of hints $\left(\sigma(\omega)^{1}, \ldots, \sigma(\omega)^{t-1}\right)$ that they had already observed.
} 
private signals that participants in groups 1, 2, 3, and 4 observed from all rounds were $\left(\sigma(A)^{1}, \sigma(B)^{2}\right) \quad, \quad\left(\sigma(A)^{1}, \sigma(A)^{2}, \sigma(B)^{3}\right) \quad, \quad\left(\sigma(A)^{1}, \sigma(A)^{2}, \sigma(A)^{3}, \sigma(B)^{4}\right) \quad, \quad$ and $\left(\sigma(A)^{1}, \sigma(A)^{2}, \sigma(A)^{3}, \sigma(A)^{4}, \sigma(B)^{5}\right)$, respectively. We name these sequences $S A_{5}, S A_{6}$, $S A_{7}$, and $S A_{8}$. Using these sequences of private signals, we designed an individual learning environment where participants in each group observed the agreeing private signals in round $t<n$ and observed the contradicting private signals in the final round $t=n$ as summarized in the third column of Table 2.

In the social learning environment, participant $i$ in group $G$ submits $\Pi(\omega)_{i, G}^{t}$, $\mu(B)_{i, G}^{t}$, and $\mu(A)_{i, G}^{t}=1-\mu(B)_{i, G}^{t}$ after observing the 'artificial' sequence of other people's predictions $\pi(\omega)$ or private signals $\sigma(\omega)$ for $n=G+1$ rounds $^{5}$. In each round $t<n$, participant $i$ in group $G$ faces a question $Q B_{G}^{t}$ that presents the other people's predictions $\pi(B)^{t}$ and asks the participant to submit $\Pi(\omega)_{i, G}^{t}$ and $\mu(B)_{i, G}^{t}{ }^{6}$. Thus, by question $Q B_{G}^{t}$, the sequences of other people's predictions that participants in

\footnotetext{
${ }^{5}$ Participants were told that several other people had already answered the same question that the participant was about to answer and that they had submitted their predictions after observing their private signals or 'their' other people's predictions in the same way as the participant would do. However, there were no 'other people' and no one had submitted predictions earlier than any participants. Instead, we presented 'artificial' sequences of other people's predictions and private signals to participants because we wanted to set up a situation where all the participants in each group observed the same sequence of other people's predictions and private signals.

${ }^{6}$ As in the individual learning environment, in round $t$, participants could also refer to the sequence of other people's predictions $\left(\pi(\omega)^{1}, \ldots, \pi(\omega)^{t-1}\right)$ that they had already observed.
} 
groups 1, 2, 3, and 4 observed from all rounds except the final round were $\left(\pi(B)^{1}\right)$, $\left(\pi(B)^{1}, \pi(B)^{2}\right), \quad\left(\pi(B)^{1}, \pi(B)^{2}, \pi(B)^{3}\right)$, and $\left(\pi(B)^{1}, \pi(B)^{2}, \pi(B)^{3}, \pi(B)^{4}\right)$, respectively. We name these sequences $S B_{1}, S B_{2}, S B_{3}$, and $S B_{4}$. On the other hand, in the final round $t=n$, participant $i$ in group $G$ faces a question $Q B_{G}^{n}$ that presents the private signal $\sigma(A)^{n}$ and asks the participant to submit $\Pi(\omega)_{i, G}^{n}$ and $\mu(B)_{i, G}^{n}$. Thus, by questions $Q B_{G}^{t}$ and $Q B_{G}^{n}$, the sequences of other people's predictions and private signals that participants in groups $1,2,3$, and 4 observed from all rounds are $\left(\pi(B)^{1}, \sigma(A)^{2}\right) \quad, \quad\left(\pi(B)^{1}, \pi(B)^{2}, \sigma(A)^{3}\right) \quad, \quad\left(\pi(B)^{1}, \pi(B)^{2}, \pi(B)^{3}, \sigma(A)^{4}\right) \quad, \quad$ and $\left(\pi(B)^{1}, \pi(B)^{2}, \pi(B)^{3},(B)^{4}, \sigma(A)^{5}\right)$, respectively. We name these sequences $S B_{5}, S B_{6}$, $S B_{7}$, and $S B_{8}$. Using these sequences of other people's predictions and private signals, we designed a social learning environment where participants in each group observe other people's agreeing predictions in round $t<n$ and observe the contradicting private signal in the final round $t=n$ as summarized in the third column of Table 2 .

In explaining the general structure of the situation in the individual and the social learning environments defined above, we presented the following description to participants. We paraphrased the state of the world as the situation where one of the boxes, either A or B, contains a piece of paper and a private signal as a hint.

"There are two boxes, A and B. One of the two boxes contains a piece of paper on which "You Win" is printed, but the other one contains nothing. The probability that Box A contains the piece of paper and that Box B contains it are 50\% and 50\%, respectively. You are not informed which box contains the piece of paper, but you can observe 'hints' or 'other people's predictions'. Your role is to submit a prediction about which box contains 
the piece of paper and its probability for several rounds based on observed sequences of hints or other people's predictions."

The question $Q A_{G}^{t}$ is presented in the beginning of round $t$ as follows.

" $Q A_{G}^{t}$ Hint: Box $\underline{A}$ contains the piece of paper.

Which box contains the piece of paper? Please submit your prediction. Then, estimate the probability that Box A contains the piece of paper and the probability that Box B contains the piece of paper and choose one of the combinations below that is the closest to your estimation. Note that this hint reports the correct answer with probability $2 / 3$ and the incorrect answer with probability $1 / 3$. Note also that the hints you observe may not always report the correct answer, but the box containing the piece of paper does not change from the first round to the final round."

After reading $Q A_{G}^{t}$, participants submit $\Pi(\omega)_{i, G}^{t}$ and $\mu(A)_{i, G}^{t}$ in each round ${ }^{7}$.

Question $Q B_{G}^{t}$ is presented in the beginning of round $t<n$ as follows, although question $Q B_{G}^{n}$ is presented in exactly the same way as $Q A_{G}^{t}$.

“ $Q B_{G}^{t}$ The other person \#t's prediction: Box $\underline{B}$ contains the piece of paper.

\footnotetext{
${ }^{7}$ Participants submit the prediction $\Pi(\omega)_{i, G}^{t}$ by choosing one of the two buttons indicating 'Box A' and 'Box B'. Participants submit the subjective posteriors $\mu(A)_{i, G}^{t}$ and $\mu(B)_{i, G}^{t}=1-\mu(A)_{i, G}^{t}$ by choosing one of the 20 combinations of probabilities that "the probability that Box A contains the piece of paper" and "the probability that Box B contains the piece of paper" from $0 \%$ to $100 \%$ at $5 \%$ intervals.
} 
Which box contains the piece of paper? Please submit your prediction. Then, estimate the probability that Box A contains the piece of paper and the probability that Box B contains it and choose one of the combinations below that is the closest to your estimation. Note that the other person \#t has submitted his/her prediction after observing other people's predictions and hints in the same way as you do."

After reading $Q B_{G}^{t}$, participants submit $\Pi(\omega)_{i, G}^{t}$ and $\mu(B)_{i, G}^{t}$ in each round.

\section{Behavioral hypotheses}

In this section, we consider the belief-updating behavior by a rational Bayesian participant in an individual and a social learning environment.

Let $P B(\omega)_{G}^{t}$ be the Bayesian posterior belief that the state of the world $\omega$ would be realized in round $t$ evaluated by participants in group $G \in\{1,2,3,4\}$. In the individual learning environment consisting of questions $Q A_{G}^{t}$, each hint that participants observe is informative, so that they can update their posterior probability in a Bayesian way in each round. For example, if participant $i$ observes the sequence of hints $\left(\sigma(A)^{1}, \ldots, \sigma(A)^{t}\right)$, the posterior probability should be:

$$
P B(A)_{G}^{t}=\operatorname{Pr}\left(A \mid \sigma(A)^{1}, \ldots, \sigma(A)^{t}\right)=\frac{\operatorname{Pr}(A) \operatorname{Pr}\left(\sigma(A)^{1}, \ldots, \sigma(A)^{t} \mid A\right)}{\operatorname{Pr}(A) \operatorname{Pr}\left(\sigma(A)^{1}, \ldots, \sigma(A)^{t} \mid A\right)+\operatorname{Pr}(B) \operatorname{Pr}\left(\sigma(A)^{1}, \ldots, \sigma(A)^{t} \mid B\right)}
$$

Given the sequence of hints we presented to participants, $P B(A)_{G}^{t}$ in each group at each round should be those summarized in the eighth column in Table 2. 
For the prediction of the state $\Pi(\omega)$, participants except group 1 at round 2 should always submit $\Pi(A)$ because they observe more $\sigma(A)$ than $\sigma(B)$.

However, in the social learning environment consisting of questions $Q B_{G}^{t}$, other people's predictions observed by participants in each round may not be informative if participants believe that other people update their beliefs in a Bayesian way as follows.

Suppose that the other person in round 1 observes $\sigma(B)^{1}$. Then, she would submit $\Pi(B)_{1, G}^{1}$ because her posterior belief is $\operatorname{Pr}\left(B \mid \sigma(B)^{1}\right)=2 / 3$. Having observed her prediction, if the other person in round 2 observes $\sigma(B)^{2}$ he would submit his prediction $\Pi(B)_{2, G}^{2}$ because his posterior belief is $\operatorname{Pr}\left(B \mid \pi(B)^{1}, \sigma(B)^{2}\right)=4 / 5$. Having observed these predictions, the other person in round 3 would submit $\Pi(B)_{3, G}^{3}$ whichever hint she observes because her posterior belief is $\operatorname{Pr}\left(B \mid \pi(B)^{1}, \pi(B)^{2}, \sigma(B)^{3}\right)=8 / 9 \quad$ if $\quad$ she observes $\quad \sigma(B)^{3} \quad$ and $\operatorname{Pr}\left(B \mid \pi(B)^{1}, \pi(B)^{2}, \sigma(A)^{3}\right)=2 / 3$ if she observes $\sigma(A)^{3}$. Because the other person in round 4 knows that the other person in round 3 always submits $\Pi(B)^{3}$, her prediction does not convey any information about the state of the world. Thus, the other person in round 4 inevitably ignores the prediction by the other person in round 3 and he submits his prediction in exactly the same way as by the person in round 3. In this way, people after round 4 ignore their predecessors' predictions and behave as if they were in round 3 if the first two people's predictions happen to correspond. Informational cascades, formulated by Bikhchandani et al. (1992), are said to occur if all the individuals in a society choose an identical action regardless of their private signals as a consequence of rational Bayesian belief updating. In our configuration of sequences of other people's 
predictions, $\pi(B)^{3}$ and $\pi(B)^{4}$ do not reflect $\sigma(B)^{3}$ and $\sigma(B)^{4}$. Thus, participants cannot update their posterior beliefs in the sequences $S B_{3}, S B_{4}, S B_{7}$, and $S B_{8}$, and informational cascades occur in these sequences. Specifically, their posterior beliefs $P B(B)_{G}^{t}$ stay constant at 0.8 in the sequences $S B_{3}$ and $S B_{4}$, and stay constant at 0.67 in the sequences $S B_{7}$ and $S B_{8}$, as summarized in the eighth column of Table 2. Note that in the sequences where informational cascades can occur, $P B(B)_{G}^{t}$ in the social learning environment is always lower than $P B(A)_{G}^{t}$ in the individual learning environment because participants no longer update their posterior beliefs.

On the other hand, other people's predictions are informative in the sequences where informational cascades cannot occur in the social learning environment because other people's predictions should reflect their observed hints. Thus, participants update their posterior beliefs in $S B_{1}$ and $S B_{2}$. In addition, hints are always informative as in the case of the individual learning environment and participants update their posterior beliefs also in $S B_{5}$ and $S B_{6}$. In such sequences, $P B(B)_{G}^{t}$ in the social learning environment is the same as $P B(A)_{G}^{t}$ in the individual learning environment because participants can update their posterior beliefs in exactly the same way as in the individual learning environment.

Given the sequence of other people's predictions we presented to participants, $P B(B)_{G}^{t}$ in each group at each round should be those summarized in the eighth column in Table 2.

For the prediction of the state $\Pi(\omega)$, participants except group 1 at round 2 should always submit $\Pi(B)$ because they observe more $\pi(B)$ than $\sigma(A)$. 


\section{Belief-updating behavior in an individual and a social learning environment}

From the predictions $\Pi(\omega)_{i, G}^{t}$ and the subjective posterior beliefs $\mu(A)_{i, G}^{t}$ and $\mu(B)_{i, G}^{t}$ that all participants in group $G$ had submitted for $n=G+1$ rounds in each of $Q A_{G}^{t}$ and $Q B_{G}^{t}$, we collected data in a total of 16 different sequences. In this section, we examine whether participants' belief-updating behaviors are consistent with the behavioral hypotheses by a rational Bayesian proposed in the previous section, and whether they differ depending on the learning environment.

The sixth column of Table 2 reports the observed proportions of predictions $\Pi(A)_{i, G}^{t}$ and $\Pi(B)_{i, G}^{t}$. When participants observed the agreeing hint, other people's agreeing prediction, or the contradicting hint in the most recent round $\left(S A_{1}, \ldots, S A_{8}\right.$ and $\left.S B_{1}, \ldots S B_{4}\right)$, more than half of them submitted $\Pi(A)_{i, G}^{t}\left(\Pi(B)_{i, G}^{t}\right)$ for question $Q A_{G}^{t}$ $\left(Q B_{G}^{t}\right)$. When participants observed other people's contradicting prediction in the most recent round (from $S B_{5}$ to $S B_{8}$ ), more than half of them submitted $\Pi(A)_{i, G}^{t}$ for question $Q B_{G}^{t}$, although they should have submitted $\Pi(B)_{i, G}^{t}$ if they rationally update their posterior beliefs. These results are inconsistent with behavioral hypotheses by a rational Bayesian in that participants in $Q A_{G}^{t}\left(Q B_{G}^{t}\right)$, except group 1 at round 2, should always submit predictions $\Pi(A)_{i, G}^{t}\left(\Pi(B)_{i, G}^{t}\right)$. In the laboratory experiments on informational cascades, such Bayesian inconsistent behaviors are often observed. In fact, Anderson and Holt (1997), Dominitz and Hung (2004), and Hung and Plott (2001) found that not all subjects submitted $\Pi(B)_{i, G}^{t}$ when they had observed exactly the same 
sequence of the other people's predictions and private signals as in $\mathrm{SB}_{6}$ and $S B_{7}{ }^{8}$. However, the proportions of $\Pi(B)_{i, G}^{t}$ in their laboratory experiments are higher than those observed in our field experiment.

For participants' subjective posteriors, the average $\mu(A)_{i, G}^{t}$ increases as the number of $\sigma(A)^{t}$ increases in $Q A_{G}^{t}$ and the average $\mu(B)_{i, G}^{t}$ increases as the number of $\pi(B)^{t}$ increases in $Q B_{G}^{t}$ as summarized in the seventh column in Table 2. However, all of the observed average subjective posteriors are lower than the Bayesian posteriors $P B(A)_{G}^{t}$ or $P B(B)_{G}^{t}$ derived in the previous section. The t-tests shown in the ninth column of Table 2 report that the observed differences between the average participants' posteriors and the Bayesian posteriors are statistically significant in almost all sequences of hints and other people's predictions. This result indicates that participants certainly use hints and other people's predictions in their probabilistic inferences, but they cannot update their posterior beliefs as efficiently as Bayesian theory assumes even when they sequentially observe informative hints in the individual learning environment. In the laboratory experiments on informational cascades, Dominitz and Hung (2004), Sasaki and Kawagoe (2006), and Stiehler (2003) observed that participants' posterior

\footnotetext{
${ }^{8}$ Anderson and Holt (1997) reported that the proportion of $\Pi(B)_{i, G}^{t}$ was 0.75 and 0.84 in the same sequence of $S B_{6}$ and $S B_{7}$. Dominitz and Hung (2004) reported that it was 0.52 and 0.80 in the same sequence of $S B_{6}$ and $S B_{7}$. Hung and Plott (2001) reported that it was 0.61 and 0.67 in the same sequence of $S B_{6}$ and $S B_{7}$. The preciseness of the private signal in their laboratory experiments is $\operatorname{Pr}(\sigma(\omega) \mid \omega)=2 / 3$, the same as the hints we used in our field experiment.
} 
beliefs were lower than the Bayesian posteriors in the same sequence of $\mathrm{SB}_{1}, \ldots, \mathrm{SB}_{8}{ }^{9}$. However, as for the case of the proportion of $\Pi(\omega)_{i, G}^{t}$, averages of $\mu(\omega)_{i, G}^{t}$ observed in their laboratory experiments were higher than those observed in our field experiment $^{10}$.

The two cells between the bold lines in the seventh column of Table 2 compare participants' average posterior beliefs in the same sequences of hints and other people's predictions between the individual and the social learning environment. As we can see, the average posterior beliefs are higher in the individual learning environment $\left(Q A_{G}^{t}\right)$ than in the social learning environment $\left(Q B_{G}^{t}\right)$. The Wilcoxon matched pair sign rank tests shown in the right-most column of Table 2 report that the observed differences between the average posterior beliefs in the individual learning environment $\left(Q A_{G}^{t}\right)$ and

${ }^{9}$ Dominitz and Hung (2004) reported that the average $\mu(B)^{t}$ was $0.61,0.68,0.74$, and 0.78 in the same sequence of $S B_{1}, S B_{2}, S B_{3}$, and $S B_{4}$, respectively. Sasaki and Kawagoe (2006) reported that the average $\mu(B)^{t}$ was $0.49,0.56,0.58$, and 0.59 in the same sequence of $S B_{5}, S B_{6}, S B_{7}$, and $S B_{8}$, respectively, and Stiehler (2003) reported that the average $\mu(B)^{t}$ was $0.47,0.58,0.63$ in the same sequence of $S B_{6}, S B_{7}$, and $S B_{8}$, respectively. The preciseness of the private information in Dominitz and Hung (2004) and in Sasaki and Kawagoe (2006) was $\operatorname{Pr}(\sigma(\omega) \mid \omega)=2 / 3$, and in Stiehler (2003) was $\operatorname{Pr}(\sigma(\omega) \mid \omega)=3 / 5$

${ }^{10}$ These differences may reflect the different design and procedure between the laboratory experiment and the field experiment. Participants in a laboratory informational cascades experiment are typically paid according to the correctness of their predictions and posterior beliefs, whereas participants in our field experiment were paid regardless of the correctness of their predictions. 
those in the social learning environment $\left(Q B_{G}^{t}\right)$ are statistically significant even in the sequences where $\mu(A)_{i, G}^{t}$ and $\mu(B)_{i, G}^{t}$ should be equal $\left(S A_{1}\right.$ and $S B_{1}, S A_{2}$ and $S B_{2}$, $S A_{5}$ and $S B_{5}$, and $S A_{6}$ and $\left.S B_{6}\right)$.

This result indicates that participants rely more on their private signals than on other people's actions even when the informativeness of both is identical. This is clear evidence of the existence of participants' cognitive biases such as overconfidence on private signals or distrust of other people's actions, which are frequently found in laboratory experiments on informational cascades (e.g., Anderson and Holt, 1997, and Nöth and Weber, 2003). These studies typically argue participants' overconfidence on the grounds of their Bayesian inconsistent behavior in discrete choice problems. However, we argue that our result is more robust than theirs because we elicited all participants' posterior beliefs in both the individual and the social learning environments and found that even the same participant, on average, submits higher posterior beliefs in the individual learning environment than in the social learning environment for all sequences.

\section{Participant's trust in other people's actions and their demographic}

\section{characteristics}

We observed that participants, on average, do not trust other people's predictions as much as their own private signals in the social learning environment. Then, what type of person is more likely to trust other people's decisions in their probabilistic inferences?

In this section, we measure each participant's degree of trust in other people's decisions and examine whether it is affected by their demographic characteristics. 
We define $\operatorname{TRUST}_{i, G}^{t}=\mu(B)_{i, G}^{t}-P B(B)_{G}^{t}$ as the degree of trust in other people's decisions for participant $i$ at round $t$ by using the data of posterior beliefs that each participant submitted in each sequence of the $Q B_{G}^{t}$ questions. Note that $T R U S T_{i, G}^{t}$ is positive if participant $i$ trusts other people's predictions and puts too much weight on $\pi_{B}^{t}$, and is negative if she distrusts them and puts too much weight on $\sigma_{A}^{t}$, compared to $P B(B)_{G}^{t}{ }^{11}$

From the data on participants' demographic characteristics, we consider participant $i$ 's age $\left(A G E_{i}\right)$, his/her gender (a dummy variable $M A L E_{i}$, which equals 1 if participant $i$ is male and 0 otherwise), his/her educational background (a dummy variable $D E G_{i}$ which equals 1 if participant $i$ has (or is expected to have) a university degree and 0 otherwise). For occupational variables, we use $M A N_{i}$, which equals 1 if participant $i$ is a manager in a private company and 0 otherwise, $\operatorname{ADMIN}_{i}$, which equals 1 if participant $i$ is an employee in an administrative position and 0 otherwise, TEA $A_{i}$, which equals 1 if participant $i$ is a teacher and 0 otherwise, and $L A W_{i}$, which equals 1 if participant $i$ is a lawyer, a CPA, or a tax accountant and 0 otherwise. We pooled data for $\operatorname{TRUST}_{i, G}^{t}$ where participants submitted the posterior beliefs $\mu(B)_{i, G}^{t}$ in each round for eight different sequences $\left(S B_{1}, \ldots, S B_{8}\right)$. Then, we

\footnotetext{
${ }^{11}$ We can check that averages of $T R U S T_{i, G}^{t}$ in all sequence $\left(S B_{1}, \ldots S B_{8}\right)$ are negative by looking at the values of $\mu(B)^{t}$ and $P B(B)_{G}^{t}$ in Table 2 as we have confirmed that participants, on average, distrust other people's actions in the previous section.
} 
regressed $\operatorname{TRUST}_{i, G}^{t}$ against $A G E_{i}, \mathrm{MALE}_{i}, \mathrm{DEG}_{i}, \mathrm{MAN}_{i}, A D M I N_{i}, T E A_{i}$, and $L A W_{i}$ using the random effects model.

Table 3 reports the result of the regression. The estimated coefficient of $M A L E_{i}$ is significantly positive, suggesting that male participants are more likely to trust other people's decisions than female participants. For the occupational variables, the estimated coefficients of $M A N_{i}, A D M I N_{i}$, and $L A W_{i}$ are significantly negative. Because we do not use a dummy variable for students, we argue that managers in a private company, employees in an administrative position, and lawyers, CPAs, and tax accountants are more likely than students to distrust other people's decisions.

\section{Participant's conformity to other people's actions and their demographic} characteristics

In this section, we measure participant's conformity to other people's decisions and examine whether it is influenced by their degree of trust in other people's decisions and their demographic characteristics.

To do this, we consider a situation where participants make decisions on the same problems with and without reference to other people's decision making. If participant $i$ makes an arbitrary decision when he cannot refer to other people's decisions, but makes the same decision as that chosen by some influential people when he can refer to their decisions, we regard such decisions as conformity to other people's decisions.

A series of questions in $Q C$ are developed to investigate whether participants' decisions on an uncertain event are influenced by authoritative people in the social learning environment. Participants were asked to predict which movie would win the $79^{\text {th }}$ Academy Award 2007 with and without reference to the result of the $64^{\text {th }}$ Golden 
Globe Award 2007, which is one of the most prestigious movie awards in the world ${ }^{12}$. If participant $i$ chooses a nonawarded movie if he/she cannot refer to the result of the Golden Globe Award and chooses the awarded movie if he/she can refer to it, we regard this participant as conforming to the authoritativeness of the award.

First, in $Q C-1$, we asked participants: "Which film will win the Best Picture award in the $79^{\text {th }}$ Academy Award 2007 among the following nominees: 'The Departed', 'Babel', 'Letters from Iwo Jima', 'Little Miss Sunshine', or 'The Queen'?”

Second, in $Q C-2$, we asked participants: “Babel' won the Best Motion Picture-Drama award in the $64^{\text {th }}$ Golden Globe Award 2007, which is often considered the preliminaries for the Academy Award. Which film will win the Best Picture award in the $79^{\text {th }}$ Academy Award 2007 among the following nominees: 'The Departed', 'Babel', 'Letters from Iwo Jima', 'Little Miss Sunshine', or 'The Queen'? We asked you the same question in $Q C-1$, but you can choose either the same or a different answer based on how you feel right now."

From the answers to these questions we define the variable $O S C A R_{i}$, which equals 1 if participant $i$ chooses anything other than "Babel" in $Q C-1$ and "Babel" in $Q C-2$ and 0 otherwise ${ }^{13}$. Then, we regress $O S C A R_{i}$ against $\operatorname{TRUST}_{i, G}^{t}, A G E_{i}$,

\footnotetext{
${ }^{12}$ Note that the answers to the questions in the experiment were accepted until February $24^{\text {th }}, 2007$, the day before the Academy Award ceremony. No participants knew the results of the Academy Award.

${ }^{13}$ Of course, some of the participant would know the result of the Golden Globe Award or they could look it up before answering $Q C-1$. Such participants might not change their answers between $Q C-1$ and $Q C-2$. However, the proportion of participants who chose "Babel" increases from $Q C-1$ to $Q C-2$ as summarized in Table 4.1.
} 
$M A L E_{i}, D E G_{i}, M A N_{i}, A D M I N_{i}, T E A_{i}$, and $L A W_{i}$ using the random effects logit model. The result of the regression is shown in the second and third columns of Table 5.

The estimated coefficient of $\operatorname{TRUST}_{i, G}^{t}$ is significantly positive. We argue that the participants who relatively trusted other people's decisions in $Q B_{G}^{t}$ are more likely to conform to authoritative people's decisions. The estimated coefficient of $A G E_{i}$ is significantly positive and that of $M A L E_{i}$ is significantly negative, implying that older and female participants are more likely to conform to authoritative people's decisions. For the occupational variables, the coefficients of $M A N_{i}, A D M I N_{i}$, and $L A W_{i}$ are significantly negative. Thus, we argue that participants working in these occupations are less likely to conform than students.

A series of questions in $Q D$ are developed to investigate whether participants' preferences are influenced by other people in the social learning environment. Participants were asked to answer whether they support the Abe cabinet in Japan with and without reference to various opinion polls. If participant $i$ changes his/her answer in accordance with the representative result of opinion polls if he/she can refer to them, we regard such participants as conforming to other people's preferences.

First, in $Q D-1$, we asked participants: "Do you support the Abe cabinet?"

Second, in $Q D-2$, we presented the results of four opinion polls, which show a decline in the approval rates of the Abe cabinet from $63 \%-70 \%$ to $39-51 \%$ in four months. Then, we asked participants: "The figure below shows changes in the approval rates of the Abe cabinet from four opinion polls. As you can see, the approval rates of the Abe cabinet have declined from September 2006 when the Abe cabinet was inaugurated. Do you support the Abe cabinet? We asked you the same question in 
$Q D-1$, but you can choose either the same answer or a different answer. Please make your decision based on how you feel right now."

From the answers to these questions we define the variable $C A B I N E T_{i}$, which equals 1 if participant $i$ answered "I support the Abe cabinet" in $Q D-1$ and answered "I do not support the Abe cabinet" in $Q D-2$ and 0 otherwise ${ }^{14}$. Then, we regress CABINET $_{i}$ against $\operatorname{TRUST}_{i, G}^{t}, A G E_{i}, \mathrm{MALE}_{i}, \mathrm{DEG}_{i}, \mathrm{MAN}_{i}, \mathrm{ADMIN}_{i}, \mathrm{TEA}_{i}$, and $L A W_{i}$ using the random effects logit model. The results of the regression are shown in the fourth and fifth columns of Table 5.

The estimated coefficient of $\operatorname{TRUST}_{i, G}^{t}$ is significantly positive. We argue that the participants who relatively trusted other people's decisions in $Q B_{G}^{t}$ are more likely to conform to other people's preferences. The estimated coefficients of $A G E_{i}$ and $D E G_{i}$ are significantly negative, implying that older and educated participants are less likely to conform. The estimated coefficients of $M A N_{i}, A D M I N_{i}$, and $T E A_{i}$ are significantly positive; thus we argue that participants working in these occupations are more likely to conform than students.

Although the degree of trust in other people's decisions in $Q B_{G}^{t}$ positively affects the conformity in both questions of $Q C$ and $Q D$, effects of participants' demographic characteristics on the conformity are not consistent for these two questions. In order to explain this inconsistency, we may have to consider the possibility that the conformity in $Q C$ and $Q D$ is caused by different mechanisms. In the literature of

\footnotetext{
14 The proportion of participants choosing "not support" increases from $Q D-1$ to $Q D-2$ as summarized in Table 4.2.
} 
social psychology, Deutsch and Gerard (1955) distinguished two types of social influence which causes the conformity. They refer to the informational social influence as the influence "to accept information obtained from another as evidence about reality." They also refer to the normative social influence as the influence "to conform with the positive expectations of another." In $Q C$, if participants make use of the result of the Golden Globe Award in predicting which movie would win the Academy Award, we can interpret that their conformity is caused by the informational social influence. On the other hand in $Q D$, if participants feel that they should comply with other people after recognizing the fact that many other people do not support the Abe cabinet, we can interpret that their conformity is caused by the normative social influence.

\section{Concluding remarks}

This study examines belief-updating behavior in individual and social learning environments. We found that participants certainly use the sequences of private signals and other people's predictions in their probabilistic inferences, but they cannot update their posterior belief as efficiently as Bayesian theory assumes because their posterior beliefs are always lower than Bayesian posteriors even when they sequentially observe informative private signals in the individual learning environment. In addition, the posterior beliefs that participants submitted in the individual learning environment are always higher than those in the social learning environment even when the informativeness of the sequences of private signals and other people's predictions is exactly the same. This observation is a clear evidence of participants' overconfidence on their own private signals or distrust of other people's actions. Furthermore, we confirmed that participant's trust in other people's actions is affected by their 
demographic characteristics. For the analysis of participant's conformity, although their trust in other people's actions positively affects the conformity for the two different situations, effects of participants' demographic characteristics on the conformity are not consistent between them. In order to explain this inconsistency, we need further investigation on the mechanism how different social influences cause the conformity in the social learning environment as Deutsche and Gerard (1955) pointed out. 


\section{Acknowledgements}

This research was supported by the Grant-in-Aid for Scientific Research 18830037 of the Ministry of Education, Culture, Sports, Science, and Technology in Japan and the 21 st Century COE program at Osaka University.

\section{References}

Anderson, L. R., Holt, C. A., 1997. Information cascades in the laboratory. American Economic Review 87, 847-862.

Bikhchandani, S., Hirshleifer, D., Welch, I., 1992. A theory of fads, fashion, custom, and cultural change as informational cascade. Journal of Political Economy 100, $992-1026$.

Deutsch, M., Gerard, H. B., 1995. A study of normative and informational social influences upon individual judgment. Journal of Abnormal and Social Psychology 51, $629-639$

Dominitz, J., Hung, A. A., 2004. Homogeneous actions and heterogeneous beliefs: experimental evidence on the formation of information cascades. Working Paper. Carnegie Mellon University.

Hung, A. A., Plott, C. R., 2001. Information cascades: replication and an extension to majority rule and conformity-rewarding institutions. American Economic Review 91, $1508-1520$. 
Nöth, M., Weber, M., 2003. information aggregation with random ordering: cascades and overconfidence. Economic Journal 113, 166-189.

Sasaki, S., Kawagoe, T., 2006. Can you believe your neighbors' behaviors? Economics Bulletin 3(11), 1-11

Stiehler, A., 2003. Do Individuals Recognize Cascade Behavior of Others? An Experimental Study. Working Paper. Max Planck Institute for Research into Economic Systems. 
Table 1. Participants' demographic characteristics

\begin{tabular}{|c|c|c|}
\hline Characteristics & & Observations \\
\hline Gender & $\begin{array}{l}\text { Male } \\
\text { Female }\end{array}$ & $\begin{array}{l}678(65.63 \%) \\
355(34.37 \%)\end{array}$ \\
\hline Age & $\begin{array}{l}20-29 \\
30-39 \\
40-49\end{array}$ & $\begin{array}{l}506(48.98 \%) \\
197(19.09 \%) \\
330(31.95 \%)\end{array}$ \\
\hline Occupation & $\begin{array}{l}\text { Managers in private company } \\
\text { Employees in an administrative position } \\
\text { Teachers } \\
\text { Lawyers, CPAs, Tax accountants } \\
\text { Students in junior colleges, universities, or } \\
\text { graduate schools }\end{array}$ & $\begin{array}{l}125(12.10 \%) \\
173(16.75 \%) \\
143(13.84 \%) \\
12(1.16 \%) \\
479(46.37 \%)\end{array}$ \\
\hline $\begin{array}{l}\text { Educational } \\
\text { Background }\end{array}$ & $\begin{array}{l}\text { Junior high school (graduated) } \\
\text { High school (dropped out) } \\
\text { High school (graduated) } \\
\text { Junior college (dropped out) } \\
\text { Junior college (graduated or will graduate) } \\
\text { University (dropped out) } \\
\text { University (graduated or will graduate) } \\
\text { Graduate school - Master's course (dropped out) } \\
\text { Graduate school - Masters' course (graduated or } \\
\text { will graduate) } \\
\text { Graduate school - Doctoral course (dropped out) } \\
\text { Graduate school - Doctoral course (graduated or } \\
\text { will graduate) } \\
\text { Not answered }\end{array}$ & $\begin{array}{c}2(0.19 \%) \\
6(0.58 \%) \\
104(10.07 \%) \\
5(0.48 \%) \\
76(7.36 \%) \\
35(3.39 \%) \\
617(59.73 \%) \\
5(0.48 \%) \\
121(11.71 \%) \\
11(1.08 \%) \\
41(3.97 \%) \\
10(0.97 \%)\end{array}$ \\
\hline
\end{tabular}


Table 2. Proportion of $\Pi(\omega)_{i, G}^{t}$, average $\mu(\omega)_{i, G}^{t}, \quad P B(\omega)_{G}^{t}$, and results of tests

\begin{tabular}{|c|c|c|c|c|c|c|c|c|c|c|c|c|c|c|c|c|}
\hline $\mathscr{\infty}$ & $\Sigma$ & $\ddot{G}$ & 5 & $\mathscr{b}$ & 5 & $\underset{d}{4}$ & 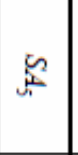 & $b_{0}$ & $\$$ & $\mathscr{b}$ & 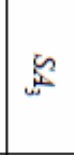 & $\mathscr{b}$ & 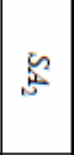 & $\mathscr{G}$ & 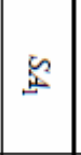 & 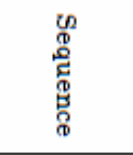 \\
\hline 's & P. & : & $\mid$ & 怘 & $\mid$ & $\mid$ & : & $\begin{array}{l}8 \\
6 \\
+\end{array}$ & ? & 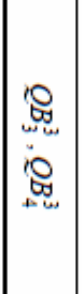 & 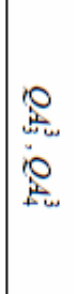 & 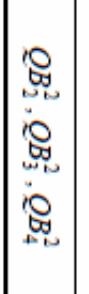 & 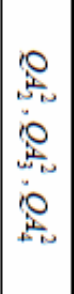 & 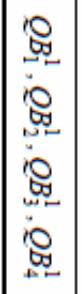 & 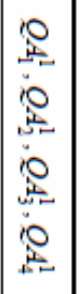 & 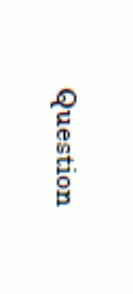 \\
\hline 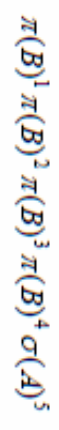 & 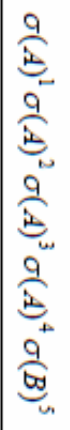 & 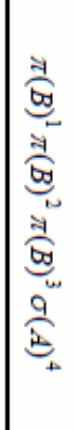 & 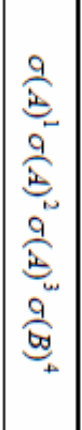 & 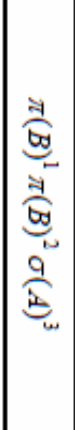 & 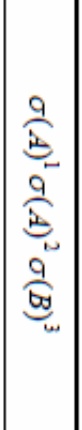 & 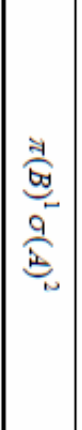 & 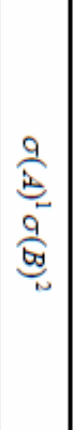 & 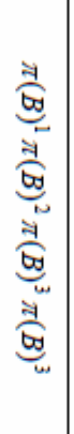 & 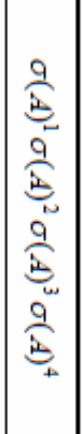 & 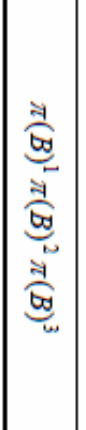 & 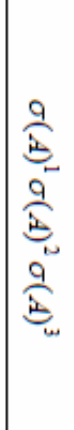 & 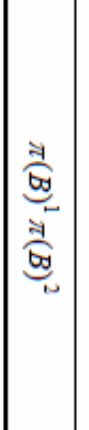 & \begin{tabular}{|l}
$\frac{9}{5}$ \\
5 \\
$\frac{9}{5}$ \\
$\frac{1}{5}$
\end{tabular} & $\underset{G}{\forall}$ & $\frac{9}{5}$ & 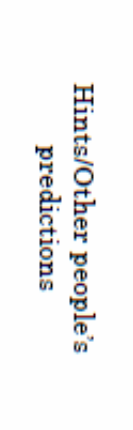 \\
\hline$\star$ & $\mapsto$ & $\omega$ & $\omega$ & 10 & 10 & $r$ & 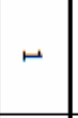 & $\star$ & $\star$ & ${ }_{i}^{\omega}$ & $\underbrace{\omega}_{i}$ & $\begin{array}{l}10 \\
\infty \\
0 \\
+\infty\end{array}$ & $\begin{array}{c}10 \\
w \\
i \\
i\end{array}$ & 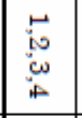 & $\begin{array}{l}\vec{H} \\
i v \\
\omega \\
i \\
i \\
\end{array}$ & 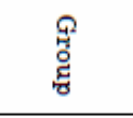 \\
\hline 战 & $\begin{array}{l}15 \\
\text { D } \\
0\end{array}$ & 谞 & 祸 & 总 & 总 & : & 용 & $\begin{array}{l}100 \\
\text { II }\end{array}$ & $\begin{array}{l}15 \\
\text { I } \\
0\end{array}$ & 号 & 임 & ઠิ & ઠี & 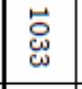 & 总 & 움 \\
\hline $\begin{array}{l}0 \\
\text { iे } \\
\text { 宫 }\end{array}$ & $\begin{array}{l}\text { 응 } \\
\text { ओे }\end{array}$ & 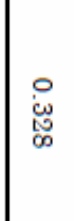 & : & i & 䀦 & $\begin{array}{l}\text { i } \\
\text { iv } \\
\text { it }\end{array}$ & 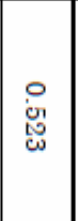 & : & 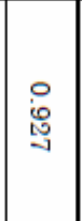 & 용 & 웅 & 思 & : & 品 & 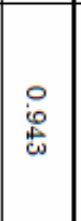 & 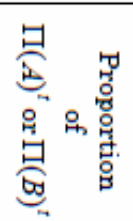 \\
\hline 道 & $\begin{array}{l}\stackrel{\circ}{O} \\
\stackrel{9}{\omega}\end{array}$ & $\begin{array}{l}\circ \\
\text { 糔 } \\
\text { 至 }\end{array}$ & $\begin{array}{l}0 \\
\text { in } \\
\text { g } \\
\text { o }\end{array}$ & 足 & 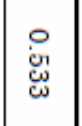 & 是 & \begin{tabular}{|l|} 
\\
0 \\
点 \\
-3
\end{tabular} & 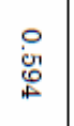 & 엄 & $\begin{array}{l}\text { 엉 } \\
\text { ํㅓㅇ }\end{array}$ & 웅 & $\begin{array}{l}\text { 응 } \\
\text { on } \\
\text { 占 }\end{array}$ & 客 & 옥 & 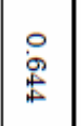 & 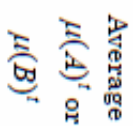 \\
\hline 용 & $\begin{array}{l}0 \\
\text { o } \\
0 \\
0\end{array}$ & 욤 & $\begin{array}{l}\circ \\
\text { o } \\
8\end{array}$ & : & :욤 & 응 & 잉 & $\begin{array}{l}\circ \\
\text { : } \\
\text { : }\end{array}$ & \begin{tabular}{l|}
$\circ$ \\
0 \\
0 \\
\multirow{4}{*}{}
\end{tabular} & $\begin{array}{l}\circ \\
\text { 영 } \\
8\end{array}$ & $\begin{array}{l}\circ \\
\text { 잉 } \\
0 \\
0\end{array}$ & $\begin{array}{l}\circ \\
\dot{0} \\
\stackrel{0}{\circ}\end{array}$ & $\begin{array}{l}\circ \\
\text { : } \\
\text { : }\end{array}$ & :욤 & $\begin{array}{l}\circ \\
\\
\\
\end{array}$ & 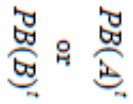 \\
\hline $\begin{array}{l}\text { II } \\
\text { ठ }\end{array}$ & 空 & $\begin{array}{l}1 \\
11 \\
0 \\
0 \\
0 \\
0\end{array}$ & 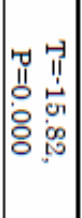 & 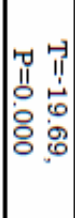 & 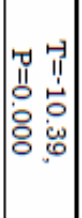 & 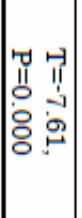 & 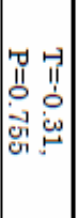 & 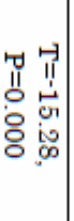 & $\mid \begin{array}{ll}0 & 1 \\
11 & 1 \\
0 & 0 \\
0 & 0 \\
0 & 0 \\
0 & j\end{array}$ & 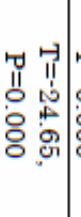 & $\begin{array}{l}0 \\
0 \\
01 \\
0.0 \\
0 \\
0 \\
0\end{array}$ & 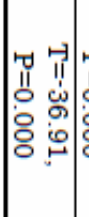 & $\mid \begin{array}{ll}0 & 1 \\
01 & 1 \\
0 & 0 \\
0 & 0 \\
0 & 0 \\
0 & 0\end{array}$ & 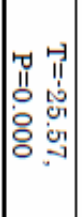 & $\mid \begin{array}{ll}0 & 0 \\
11 & 1 \\
0 & 0 \\
0 & 0 \\
0 & 0 \\
0 & 0\end{array}$ & 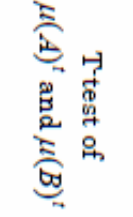 \\
\hline$\frac{\mathrm{O}}{\mathrm{N}}$ & 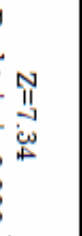 & 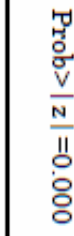 & $\prod_{i=1}^{N}$ & $\frac{\mathrm{v}}{\mathrm{N}}$ & $\begin{array}{l}N \\
11 \\
0 \\
1 \\
4\end{array}$ & 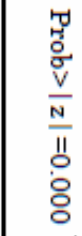 & $\begin{array}{l}\text { N1 } \\
\text { In } \\
8\end{array}$ & $\begin{array}{l}\frac{T}{0} \\
\frac{\dot{O}}{\sigma} \\
\frac{\mathrm{V}}{\mathrm{N}} \\
\frac{11}{0} \\
\dot{\delta}\end{array}$ & $\frac{1}{10}$ & 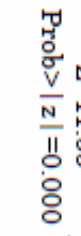 & 帒 & 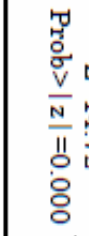 & 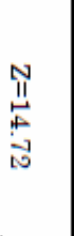 & $\begin{array}{l}\frac{T}{0} \\
\frac{\sigma}{\sigma} \\
\frac{\mathrm{V}}{\mathrm{N}} \\
1 \\
0 \\
\dot{0}\end{array}$ & 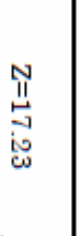 & 象 \\
\hline
\end{tabular}


Table 3. Participant's trust in other people's actions and their demographic characteristics

\begin{tabular}{|l|r|r|}
\hline Dependent variable & TRUST $_{i, G}^{t}$ & $\mathrm{p}>|\mathrm{z}|$ \\
\hline$A G E_{i}$ & 0.1385 & 0.180 \\
\hline$M A L E_{i}$ & 3.1803 & 0.010 \\
\hline$D E G_{i}$ & 1.1803 & 0.375 \\
\hline$M A N_{i}$ & -4.3092 & 0.086 \\
\hline$A D M I N_{i}$ & -5.1482 & 0.032 \\
\hline TEA $_{i}$ & 0.0099 & 0.962 \\
\hline LAW $_{i}$ & -9.0024 & 0.078 \\
\hline Constant & -23.8344 & 0.000 \\
\hline Number of observations & 3577 \\
\hline Number of participants & \multicolumn{2}{|c|}{1023} \\
\hline $\mathrm{R}^{2}$ & \multicolumn{2}{|c|}{0.0125} \\
\hline Wald Chi2(7) & \multicolumn{2}{|c|}{0.010 .48} \\
\hline Prob>Chi2 & \multicolumn{2}{|c|}{} \\
\hline
\end{tabular}


Table 4.1. The proportion of answers in $Q C-1$ and $Q C-2$

\begin{tabular}{|c|c|c|}
\hline Answers & $Q C-1$ & $Q C-2$ \\
\hline Babel & 0.2197 & 0.3359 \\
\hline The Departed & 0.0949 & 0.0842 \\
\hline Letters from Iwo Jima & 0.5537 & 0.4695 \\
\hline Little Miss Sunshine & 0.0697 & 0.0591 \\
\hline The Queen & 0.0620 & 0.0513 \\
\hline
\end{tabular}

Table 4.2. The proportion of answers in $Q D-1$ and $Q D-2$

\begin{tabular}{|l|r|r|}
\hline Answers & \multicolumn{1}{|l|}{$Q D-1$} & \multicolumn{1}{l|}{$Q D-2$} \\
\hline Support & 0.3040 & 0.2865 \\
\hline Not support & 0.6960 & 0.7135 \\
\hline
\end{tabular}


Table 5. Participant's conformity to other people's actions and their demographic characteristics

\begin{tabular}{|c|c|c|c|c|}
\hline Dependent variable & OSCAR $_{i}$ & $\mathrm{p}>|\mathrm{z}|$ & CABINET $_{i}$ & $p>|z|$ \\
\hline $\operatorname{TRUST}_{i, G}^{t}$ & 0.0086 & 0.096 & 0.020 & 0.028 \\
\hline$A G E_{i}$ & 0.0420 & 0.094 & -0.1651 & 0.000 \\
\hline$M A L E_{i}$ & -0.6217 & 0.036 & -0.0952 & 0.846 \\
\hline$D E G_{i}$ & -0.5032 & 0.125 & -1.0434 & 0.022 \\
\hline$M A N_{i}$ & -1.9746 & 0.001 & 2.7566 & 0.003 \\
\hline ADMIN $_{i}$ & -1.5848 & 0.007 & 3.2674 & 0.000 \\
\hline$T E A_{i}$ & -0.2725 & 0.590 & 1.9052 & 0.015 \\
\hline$L A W_{i}$ & -2.0570 & 0.062 & -17.8440 & 1.000 \\
\hline Constant & -3.5477 & 0.000 & -1.5717 & 0.131 \\
\hline $\begin{array}{l}\text { Number of } \\
\text { observations }\end{array}$ & \multicolumn{2}{|c|}{3577} & \multicolumn{2}{|c|}{3577} \\
\hline $\begin{array}{l}\text { Number of } \\
\text { participants }\end{array}$ & \multicolumn{2}{|c|}{1023} & \multicolumn{2}{|c|}{1023} \\
\hline Wald Chi2(8) & \multicolumn{2}{|c|}{35.44} & \multicolumn{2}{|c|}{28.81} \\
\hline Prob $>$ Chi2 & \multicolumn{2}{|c|}{0.0000} & \multicolumn{2}{|c|}{0.0003} \\
\hline
\end{tabular}

